\title{
Desarrollo del entorno personal de aprendizaje: valoración de una experiencia con estudiantes universitarios
}

\section{Development of Personal Learning Environment: assessment of an experi- ence with university students}

\author{
José Antonio García Martínez* \\ Manuel Arturo Fallas Vargas \\ Alejandra Gamboa Jiménez \\ Universidad Nacional, Costa Rica \\ (Rec.: octubre de 2016 - Acept.: noviembre de 2016)
}

\begin{abstract}
Resumen
En este trabajo se muestra los resultados de un estudio derivado de una experiencia cuyo objetivo es analizar el desarrollo del entorno personal de aprendizaje (PLE) de estudiantes universitarios de primer año. El colectivo participante queda compuesto por 96 estudiantes matriculados en el curso de Recursos Tecnológicos de Bachillerato en Orientación de la Universidad Nacional (Costa Rica). Tanto el diagnóstico inicial como la valoración final se obtienen por medio de un cuestionario en línea. De los principales resultados se deriva el poco desarrollo del PLE del estudiantado en el momento de ingresar a la universidad, la valoración positiva que realizan de desarrollo del PLE al finalizar el curso, así como la alta previsión de uso de las diferentes herramientas tanto a nivel personal como profesional.
\end{abstract}

Palabras clave: entorno personal de aprendizaje, estudiantes universitarios, tecnologías de la información y comunicación.

\begin{abstract}
This work presents the results of a study derived from an experience whose objective is to analyze the development of personal learning environment (PLE) in first-year university students. The participant group is composed of 96 students enrolled in the course of Technological Resources of Bachelor's Degree in Guidance of the Universidad Nacional de Costa Rica. The first diagnosis as well as the final assessment are obtained through an online questionnaire. The main results show the student body's low development of PLE at the time of joining university, the positive assessment made about the development of PLE at the end of the course, as well as the high forecast use of the different tools, both at personal and professional level.
\end{abstract}

Keywords: personal learning environment, university students, information and communication technologies.

\footnotetext{
* Correspondencia a: José García Martínez. Centro de Investigación en Docencia y Educación. División de Educaciòn para el Trabajo. Universidad Nacional, Heredia. Costa Rica. E-mail: jose.garcia.martinez@una.cr.
} 


\section{Introducción}

A diferencia de otros países, en Costa Rica, la Orientación es una disciplina y una profesión que posee explícitamente una carrera universitaria para poder ejercer en el mundo laboral actual. En este contexto, el colectivo de profesionales de la orientación se encuentra ante diferentes desafíos con poblaciones y escenarios diversos y complejos tales como el sector educativo, sistema de justicia y paz, técnico, geriátrico, organizaciones no gubernamentales, entre otros. En este sentido, tiene que ver principalmente con el desarrollo personal-social, profesional, vocacional, laboral, familiar y comunitario de las personas o de los colectivos con los cuales se realiza labor orientadora.

Lo anterior ubica el accionar del profesional en orientación ante una gran gama de dimensiones donde se presentan retos cotidianos en su quehacer. El uso de las tecnologías de la información y la comunicación (TIC) se torna en un recurso aliado más para desempeñar el trabajo y dar sentido a las teorías que fundamentan la labor orientadora, así como para fortalecer el personal learning environment [entorno personal de aprendizaje] (PLE).

Es por ello que, de acuerdo a Muñoz-Carril y González-Sanmmamed (2015), "el binomio orientación-tecnología suscita implicaciones importantes en la consideración del rol de los profesionales de la orientación" (p. 449), tanto en los procesos propios de orientación como en las herramientas utilizadas, aspectos que deben condicionar la formación que reciben los profesionales desde las universidades.

En el caso de la Universidad Nacional de Costa Rica (UNA), el curso de Recursos Tecnológicos para la Orientación se ofrece en el primer nivel del plan de estudios de bachillerato de la carrera de Orientación. Este curso brinda al colectivo discente los conocimientos teórico-prácticos que le permitirán hacer uso de diversas herramientas tecnológicas y aplicarlas tanto en su ejercicio profesional como en el proceso de formación a lo largo de la vida. Se hace énfasis en la elaboración de materiales y utilización de las TIC, especialmente de la web 2.0, que puedan facilitar la mediación de procesos orientadores (UNA, 2006).

El curso promueve la construcción de aprendizajes a través del uso de herramientas tecnológicas, con las cuales el estudiantado logre crear, desde una perspectiva profesional orientadora, productos innovadores, creativos y de valor tanto personal como profesional. El abordaje metodológico del curso se lleva a cabo desde el aprendizaje por proyectos, donde el colectivo discente realiza diferentes proyectos tanto a nivel individual como colectivo en base a lecturas, temas, artículos, entre otros, relacionados con el ámbito de la orientación.
El objetivo general del estudio es analizar el desarrollo del PLE del estudiantado, en dos momentos, al ingresar a la universidad y después de realizar el curso de Recursos Tecnológicos para la Orientación.

Específicamente se pretende, en primer lugar, diagnosticar las herramientas que utiliza el estudiantado y su frecuencia de uso al entrar en la Universidad. En segundo lugar, identificar la valoración que el estudiantado hace de las herramientas utilizadas a lo largo del curso, así como el grado de dificultad de las mismas. En tercer lugar, identificar la previsión de uso a nivel personal y profesional que hará el estudiantado de los diferentes recursos utilizados durante el curso $y$, por último se pretende describir la valoración que el estudiantado hace del curso, en términos generales, así como la percepción en cuanto al desarrollo del PLE.

Son múltiples las investigaciones realizadas en torno a los PLE (Cabero \& Marín, 2011; Castañeda \& Soto, 2010; Venkatesh, Croteau y Rahab, 2014; García-Martínez \& González-Sanmamed, en prensa) que se bifurcan en torno a dos temas; por un lado, aquellas que se centran en el aprendizaje del estudiantado, y, por otro, aquellas con una posición más tecnológica que tratan de identificar las herramientas más utilizadas dentro de los PLE.

De las investigaciones en el ámbito internacional que tienen como objetivo el desarrollo de los PLE, Castañeda y Soto (2010) presentan un estudio con alumnos universitarios, concluyendo, por un lado, la falta de experiencia en el uso de las TIC (aparte de las redes sociales) antes de su entrada en la universidad, y, por otro, la valoración positiva sobre algunas de las herramientas. Adicionalmente, Marín, Lizana y Salinas (2014) desarrollan una estrategia para integrar aprendizajes en la universidad a través de los PLE, donde se observa la valoración positiva del estudiantado en aspectos como la estrategia, relevancia, utilidad de las herramientas y posibilidad de utilizarlas tanto a nivel personal como profesional.

En el ámbito costarricense se lleva a cabo una investigación (García-Martínez \& González-Sanmamed, en prensa), donde se analiza el PLE de docentes en formación, específicamente, respecto a las herramientas para buscar información. En este sentido, se detecta una notable necesidad del desarrollo del PLE, así como el desconocimiento de ciertas herramientas y el poco uso que hacen de las mismas.

\section{Marco Teórico}

\section{Concepto de PLE}

No cabe duda que el PLE es uno de los temas referentes a tecnología educativa que más auge está cobrando en los últimos años. Una prueba de ello es la conferencia anual "PLE Conference" que se desarrolla 
desde el 2010 en diferentes países (García-Martínez \& González-Sanmamed, en prensa).

Se parte de la base de que todas las personas tienen un PLE, sean conscientes o no de ello. Este PLE no tiene por qué depender solo de herramientas tecnológicas; sin embargo, teniendo en cuenta el auge de recursos adaptados para la educación (especialmente herramientas de la web 2.0) en los últimos años, así como el curso en el cual se enmarca este estudio, se hará especial énfasis las mencionadas herramientas tecnológicas. Así, en el presente trabajo el PLE será definido como "(...) el conjunto de herramientas, fuentes de información, conexiones y actividades que cada persona utiliza de forma asidua para aprender" (Adell \& Castañeda, 2010, p. 23). Es decir, todo aquello que utiliza una persona para su aprendizaje desde una perspectiva holística (García-Martínez \& González-Sanmamed, en prensa).

Este concepto no está exento de discusión, autores como Anderson (como se citó en Adell, 2009), señalan algunas barreras para que se puedan adoptar los PLE en la formación. Entre estas que destacan:

- El nivel básico de la competencia tecnológica de muchos estudiantes universitarios y profesionales.

- La complejidad a la hora de conciliar los aspectos dirigistas y prescriptivos de la educación formal con las estrategias y prácticas de aprendizaje informal que nutren a los PLE.

\section{Componentes del PLE}

Al igual que ocurre con la terminología, también se ha discutido sobre la estructura o las partes que contempla un PLE, y lo único que parece estar claro es, de acuerdo con Castañeda y Adell (2013), "que no existen ni herramientas, ni estrategias, ni mecanismos que puedan ser considerados como exclusivos de una única parte del PLE. (...)" (p. 18). Es decir, que cualquiera de estos tres aspectos puede reflejarse en una sola parte o en varias, tanto de manera aislada como simultánea.

Algunos teóricos como Wheeler (como se citó en Marín et al., 2014), consideran que las funciones que cumplen los PLE se resumen en gestionar la información, la creación de contenidos y la conexión con otras personas, concordando así con la estructura que proponen Castañeda y Adell (2011). Esta se divide en las siguientes partes:

1. Herramientas y estrategias de lectura: las fuentes de información a las que accedo que me ofrecen dicha información en forma de objeto o artefacto (mediatecas).

2. Herramientas y estrategias de reflexión: los entornos o servicios en los que puedo transformar la in- formación (sitios donde escribo, comento, analizo, recreo, público).

3. Herramientas y estrategias de relación: entornos donde me relaciono con otras personas de/con las que aprendo. (p. 89)

Actualmente tenemos acceso a una gran cantidad de herramientas que pueden incluirse en nuestros PLE. Attwell (2007) muestra algunas de las que utiliza en su entorno personal de aprendizaje: procesador de textos, audio para podcasts, weblog personal, motores de búsqueda, sistemas de gestión de contenidos, etc.

Otras investigaciones (Cabero \& Marín, 2011; Castañeda \& Soto, 2010; Prendes, Castañeda, Ovelar \& Carrera, 2014; Ruiz, Sánchez \& Gómez-García, 2013) muestran las principales herramientas que utilizan tanto el colectivo de estudiantes como de docentes en sus PLE, existiendo coincidencia en gran parte de los recursos utilizados. Estos recursos son los que se han utilizado a lo largo del curso y que se exponen en la metodología (figura 1).

\section{Algunas teorías que fundamentan los PLE}

El PLE no es una teoría de la enseñanza y el aprendizaje, ni se deriva de ellos (Castañeda \& Adell, 2013). No obstante, con la incorporación de las TIC a procesos tanto formales como informales de educación, se han adaptado ciertas teorías ya existentes para fundamentar el uso y las posibilidades que estas ofrecen, tal es el caso del constructivismo. Por otro lado, han surgido teorías nuevas como el conectivismo o la heutagogía, u otras para los PLE, específicamente, como la teoría LaaN. A continuación, todas estas teorías serán descritas.

\section{Constructivismo}

Como se ha indicado con anterioridad, con la llegada del concepto PLE se retoma la idea de descentralizar las estrategias didácticas de la institución y centrarlas en el colectivo de estudiantes. Este aspecto que tiene que ver con la educación flexible y el aprendizaje abierto, donde "el usuario tiene elección, tiene libertad de maniobra, tiene control sobre la forma en que aprende" (Salinas, 2013, p. 53). Sin embargo, este aspecto no es exclusivo del PLE, prueba de ello es el cuadrante de paradigmas pedagógicos para el e-learning propuesto por Coomey y Stephenson (2001) donde se muestran las diferentes características según se otorgue mayor o menor peso al docente o al estudiante.

De acuerdo con Salinas (2013), lo anterior va ligado (más aún tratándose de estudiantes universitarios) a estrategias didácticas donde se incluyen tareas, actividades y experiencias abiertas y centradas en el estudiantado. Es aquí donde emerge de forma destacada el aprendizaje desde el trabajo colaborativo, con mayor peso de la investigación y el descubrimiento y funda- 
mentado en teorías constructivistas.

Se genera así un nexo de unión entre las prácticas desarrolladas con los PLE y la teoría constructivista de la enseñanza y el aprendizaje (Castañeda \& Adell, 2013), entendiendo que el constructivismo se basa en un proceso de construcción de aprendizaje activo por parte de la persona aprendiente, y donde el proceso de enseñanza facilita esta construcción (Duffy \& Cunningham, 1996).

Todos estos aspectos mencionados pueden verse beneficiados con ciertas herramientas tecnológicas, actividades y mecanismos integrantes de los PLE. Este conjunto es utilizado por el estudiantado de la carrera de Orientación bien para acceder a información (destacando su participación activa); bien para reflexionar sobre esta misma y generar a su vez contenidos novedosos en los nuevos espacios que ciertas herramientas generan (donde puede desarrollarse la criticidad y el trabajo colaborativo tanto dentro como fuera del aula de clase); bien para compartir con las demás personas, generando mayor aprendizaje.

\section{Conectivismo}

Si bien el conectivismo ha recibido críticas y no deja de generarse controversia en torno a esta teoría creada por Siemens (Castañeda \& Adell, 2013, quizá sea una de las teorías que mayor impacto ha tenido en la integración de las TIC en la educación. Así mismo, se aproxima a generar una base teórica para los PLE, donde tal vez por su generalidad, los principios propuestos son adaptables a las prácticas que se generan con los PLE, al igual que en el constructivismo. Estos principios suponen que:

- El aprendizaje y el conocimiento dependen de la diversidad de opiniones.

- El aprendizaje es un proceso de conectar nodos o fuentes de información especializados.

- El aprendizaje puede residir en dispositivos no humanos.

- La capacidad de saber más es más crítica que aquello que se sabe en un momento dado.

- La alimentación y mantenimiento de las conexiones es necesaria para facilitar el aprendizaje continuo.

- La habilidad de ver conexiones entre áreas, ideas y conceptos es una habilidad clave.

- La actualización (conocimiento preciso y actual) es la intención de todas las actividades conectivistas de aprendizaje.

- La toma de decisiones es, en sí misma, un proceso de aprendizaje. El acto de escoger qué aprender y el significado de la información que se recibe, es visto a través del lente de una realidad cambiante. Una decisión correcta hoy, puede estar equivocada mañana debido a alteraciones en el entorno informativo que afecta la decisión (Siemens, 2004, p. 7).

Esta teoría coloca al aprendiente como piedra angular del aprendizaje, y se hace eco "(...) tanto de artefactos culturales que contienen información valiosa para la recreación del conocimiento, como de personas que median, transforman y relacionan esa u otra información y otros conocimientos" (Castañeda \& Adell, 2013, p. 32). A su vez, también se desprende del conectivismo, la importancia de que la persona conozca (a través de la propia toma de decisiones) su PLE, la importancia de su estructura y el aprendizaje que genera en ella misma.

\section{Teoría LaaN}

La teoría Learning as a Network (LaaN) [aprendizaje como una red] es según Castañeda y Adell (2013) "(...) un intento de elaborar una fundamentación teórica sobre el aprendizaje y la enseñanza cuya puesta en acción sea la construcción y el enriquecimiento del propio PLE (...)" (p. 38). Quizá, para salir del intento necesita mayor discusión y socialización, así como desarrollar una propuesta en el marco de trabajos de investigación.

Esta teoría se alimenta de otras como el conectivismo, la teoría de la complejidad y el aprendizaje de doble bucle, con las cuales converge en una serie de aspectos. Sin embargo, según Chatti (2013), su principal distinción resulta tanto del énfasis que se da al estudiantado como de la creación de una personal knowledge network (red personal de conocimiento o PKN), donde el estudiantado puede gestionar su aprendizaje integrando las diferentes herramientas y posibilidades, desde el ámbito formal, informal o no formal (Salinas, 2013).

\section{La importancia de los PLE en la profesión orienta- dora}

Tomando en cuenta los diversos escenarios laborales, así como a las demandas del ejercicio profesional de orientación en los respectivos ámbitos de trabajo, las TIC se han convertido en aliadas fundamentales en respuesta a los procesos orientadores de los distintos grupos etarios con los cuales se trabaja. No obstante, al igual que otros gremios "la orientación tiene la urgente necesidad de afrontar las grandes transformaciones tecnológicas, económicas y sociales que están operando (...)" (Pantoja \& Campoy, 2001, p.99).

Dentro de la disciplina, la incorporación de las TIC y el desarrollo del PLE cobran mayor pertinencia en las áreas de orientación vocacional y profesional, a lo cual contribuye el análisis exhaustivo que realiza Álvarez (1995). Este autor argumenta la importancia del pro- 
ceso vocacional debido a su carácter técnico y profesional, al realizar las intervenciones orientadoras, y a la preparación para la vida (y a lo largo de la misma) de la persona orientada.

El colectivo orientador se encuentra ante varios retos. Por un lado, el personal-profesional debe enfrentar un mundo en constante cambio $y$, por otro, las propias personas orientadas deben afrontar la evolución constante de la sociedad en un mundo vorazmente globalizado.

La tecnología ha impactado, como en muchas otras áreas, el ámbito educativo, donde la disciplina orientadora no es la excepción, especialmente en relación con los planteamientos del PLE, siendo pertinentes las premisas relacionadas con la orientación vocacional y profesional, aunque como indica Rodríguez (1998), "La orientación profesional del futuro deberá recoger y ampliar lo hasta ahora hecho, pero deberá iniciar caminos innovadores redefiniendo su marco de referencia y los contextos y objetivos que demanda una nueva era" (p.31).

Aunado a lo anterior, Pantoja y Campoy (2001) señalan que la persona profesional en orientación debe desarrollar ciertos roles y funciones relacionados con las TIC. Entre ellos destacan: conocer los principales recursos para poder implementarlos en su labor, diseñar programas de intervención a través de las TIC y ayudar a las personas orientadas a utilizarlas $y$, por ende, a que desarrollen sus propios PLE.

Si bien es cierto que las tecnologías pueden ayudar en el quehacer profesional, aspectos como la gran cantidad de información a la que se tiene acceso o el surgimiento vertiginoso de nuevas herramientas, especialmente de la web 2.0, pueden suponer limitaciones para el uso de las mismas. Sumado a esto y de acuerdo con Nogueira-Pérez, Fernández-Sestelo, Muradás-López y Porta-Marín (2005), la carencia de una formación especializada en TIC para la orientación, así como la falta de tradición en la integración de recursos tecnológicos en procesos orientadores, suponen obstáculos para la adquisición de nuevos roles o competencias al respecto.

Es sustantivo que, dentro de la profesionalidad que va adquiriendo la persona orientadora en formación, esta genere las habilidades que la inclinen a plantearse la innovación y renovación constante de su trabajo. Adicionalmente, ha de tener en cuenta la primacía de los aspectos éticos en el uso de las herramientas, así como su evaluación y adaptación al contexto en un sentido amplio. Del mismo modo, esta persona profesional de la orientación ha de garantizar el bienestar de las personas (National Career Development Association [NCDA], como se citó en Sobrado \& Cortés, 2009).

Si bien los citados aspectos del código de ética de la
NCDA no se encuentran recogidos por el Código de Ética del Colegio de Profesionales en Orientación de Costa Rica, sirven de referencia para aplicar a las prácticas profesionales del colectivo de trabajadores que nos ocupa.

\section{Metodología}

De cara al cumplimiento de los objetivos propuestos, el enfoque metodológico del estudio es cuantitativo (Hernández, Fernández \& Baptista, 2010). Su diseño es ex post-facto y su alcance es exploratorio-descriptivo, ya que permite dar respuesta al estado presente de una situación educativa concreta (Bisquerra, 2014), en relación al PLE del estudiantado universitario, que ha sido poco estudiado en el ámbito costarricense.

\section{Participantes}

Para el presente estudio, la población quedó compuesta por el colectivo de estudiantes de primer año que cursó la asignatura de Recursos Tecnológicos para Orientación durante los años 2015 y 2016. Esta asignatura pertenece al Bachillerato de Orientación que se imparte en la UNA, concretamente en el Campus Omar Dengo. Este curso se imparte en los laboratorios de cómputo del Centro de Investigación y Docencia en Educación (CIDE) y se divide anualmente en tres grupos.

A lo largo de estos dos años, se matricularon en el curso un total de 119 estudiantes, de los cuales 96 participaron del estudio $(n=96)$, concretamente, 58 estudiantes en 2015 y 38 en 2016. La edad del colectivo discente que participó en el estudio osciló entre los 17 y los 35 años (Md=18; $\bar{X}=19.4 ; \sigma=3,1)$. En el grupo participante, 69 estudiantes eran mujeres (71,9\%), mientras que 27 eran hombres $(28,1 \%)$.

\section{Instrumento}

Como instrumento de recolección de datos se utilizaron dos cuestionarios en línea. El primero de ellos para diagnosticar las herramientas que el estudiantado utilizaba dentro de sus PLE hasta el inicio del curso. El mismo se facilitó a través de un sistema de gestión de aprendizaje (aula virtual) al cual el colectivo de estudiantes tuvo acceso. El cuestionario quedó compuesto por dos bloques (ambos con preguntas cerradas); el primero de ellos con preguntas demográficas, y el segundo con una escala tipo Likert, donde se ofrecía un listado de herramientas para marcar la frecuencia de uso. Dicha escala constaba de tres opciones: "frecuentemente", "algunas veces" y "nunca". Aquellas personas que indicaban no haber utilizado el recurso tenían la opción de señalar "nunca pero la conozco" o "nunca porque la desconozco".

En la última sesión de curso se facilitó otro cuestionario en línea; el mismo constó de tres bloques. El primero quedó compuesto por una escala donde el estudian- 
tado valoró las herramientas utilizadas a lo largo del curso a través de cuatro opciones de respuesta: "muy insatisfecho", "satisfecho", "insatisfecho" y "muy insatisfecho". Un segundo bloque quedó constituido por otra escala de tres opciones de respuesta respecto al grado de dificultad de las herramientas. Por último, el tercer bloque contenía la previsión de uso de los recursos utilizados, así como dos preguntas abiertas para que el estudiantado opinase sobre la metodología utilizada y la percepción sobre el desarrollo de su PLE a lo largo del curso.

Una vez recogidos los datos en una hoja de cálculo y teniendo en cuenta que los instrumentos eran en línea, se exportaron al paquete estadístico SPSS 19.0 para la realización de los distintos análisis desde una perspectiva de estadística descriptiva, obteniendo las diferentes frecuencias, gráficos, así como estadísticos que describen la muestra.

\section{Proceso}

El curso, como viene siendo habitual, inició en febrero y finalizó en junio, teniendo una duración de 17 semanas, de las cuales 15 fueron presenciales y dos virtuales. En la figura 1, se observan las distintas fases en las cuales se estructuró el curso. Cabe resaltar que las distintas actividades y proyectos fueron elaborados en base a lecturas o información relacionada con la disciplina orientadora, donde el estudiantado decidió qué recursos utilizar en cada caso. Igualmente, de manera transversal (a lo largo de todo el curso), se llevó a cabo un proceso de análisis de la importancia del desarrollo del PLE para la persona profesional en orientación, así como su vinculación con el contexto actual de trabajo y los cambios acaecidos en los últimos años.
En la tercera fase, se mostraron contenidos y se facilitaron recursos para la gestión y creación de contenidos, donde el estudiantado, según su preferencia, pudo incorporar otras herramientas distintas dentro de sus PLE.

La cuarta fase versó sobre aquellos recursos para compartir, trabajar de manera colaborativa y aprender, con otros usuarios en red. Esta fase se desarrolló en el marco de un proyecto final en grupo.

La quinta y última fase se refirió al proceso de evaluación, donde el colectivo discente realizó la evaluación del curso y de las herramientas vistas a lo largo del mismo, además de generar un espacio de discusión en torno al desarrollo de sus propios PLE.

\section{Resultados}

\section{Situación inicial del estudiantado}

Durante la primera sesión del curso, se realizó un diagnóstico a través de un cuestionario en línea con el objeto de conocer las herramientas que el estudiantado utilizaba en su PLE, así como la frecuencia de uso de las mismas. Como se observa en la figura 2, las herramientas más utilizadas por el estudiantado eran las redes sociales (Facebook, Instagram), los buscadores no especializados (Google), los recursos para hacer presentaciones (Power Point), algunos procesadores de texto (Word) y el correo electrónico (Hotmail y Gmail). Las herramientas que el colectivo discente menos utilizaba eran los mundos virtuales, seguido de los sistemas de gestión de aprendizaje (aulas virtuales) y los lectores RSS, así como los podcasts y los cuadernos de notas en línea.

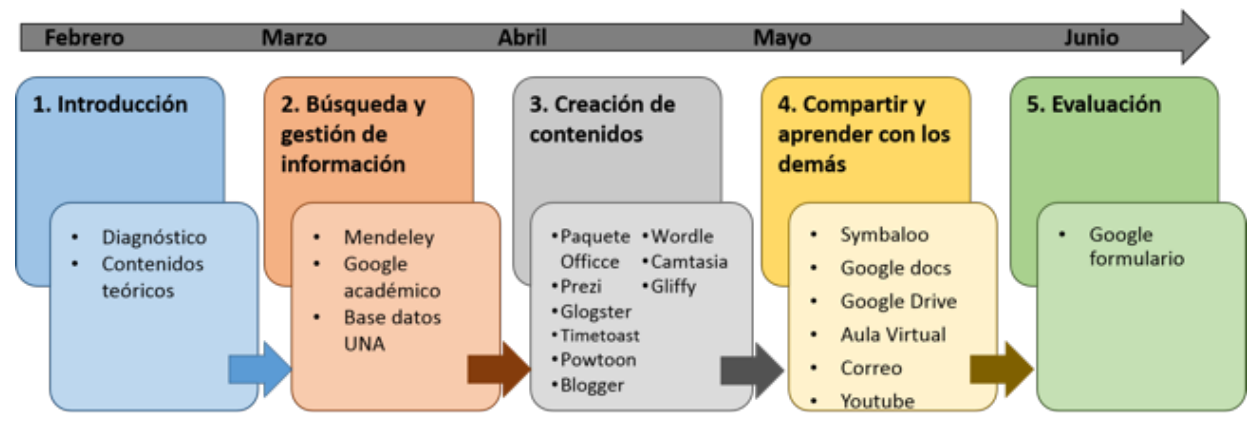

Figura 1. Fases y temporalización del curso

En la primera fase, se realizó un diagnóstico y el colectivo de estudiantes discutió sobre sus propios PLE. Con esta base inicial, se desarrollaron contenidos teóricos como el concepto, componentes y principales teorías en torno a los PLE.

Posteriormente, se desarrolló la segunda fase con herramientas para buscar y gestionar la información, resaltando la presencia de un especialista para explicar el sistema de búsqueda de la UNA.
Las personas que declararon no utilizar nunca las herramientas (como se observa en la figura 3) aludieron en su gran mayoría al desconocimiento de las mismas. Este es el caso del mundo virtual (97.8\%) y los lectores $\mathrm{RSS}(97.7 \%)$, al igual que los cuadernos de notas (92.3\%), los podcast (85.0\%), el aula virtual (83.3\%) y las herramientas para la creación colaborativa de contenidos (82.3\%). Con respecto a otras herramientas como los blogs y las wikis, el estudiantado indicó conocerlas a pesar de no utilizarlas. 


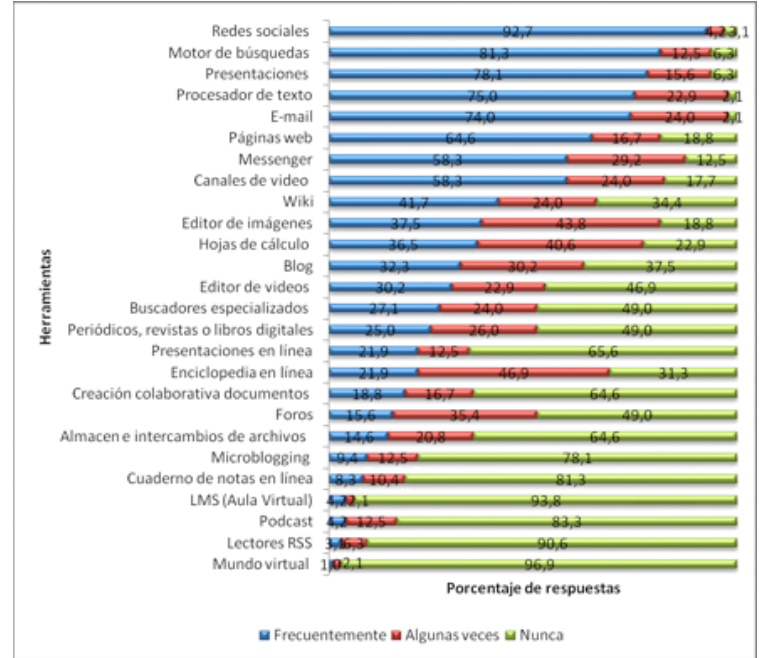

Figura 2. Frecuencia de uso de las herramientas que utilizaba el estudiantado al inicio del curso.

\section{Valoración de las herramientas utilizadas durante el curso}

A continuación, se muestra la valoración que el estudiantado realizó de los recursos utilizados a lo largo del curso. Para una presentación más adecuada, las herramientas han sido divididas en aquellas que se utilizaron para buscar y gestionar la información, para crear y gestionar contenidos y para compartir y aprender con los demás usuarios, respetando así la estructura del PLE.

Como se observa en la figura 4 , el colectivo discente valoró muy favorablemente las herramientas para la gestión de referencias (Mendeley), así como las bases de datos de la UNA y los buscadores especializados. En los tres casos, casi el total (98\% aprox.) del estudiantado indicó estar "muy satisfecho" o "satisfecho", y solamente el $2 \%$ restante declaró estar "insatisfecho" o "muy insatisfecho".

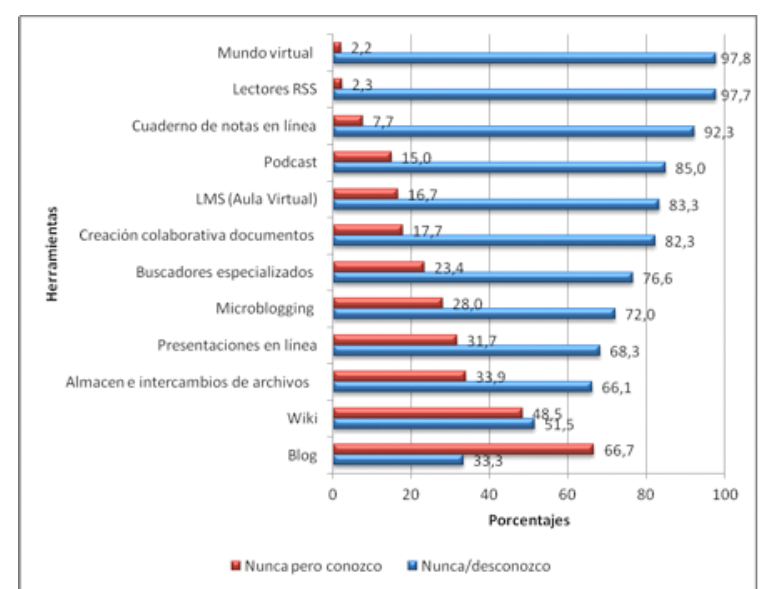

Figura 3. Herramientas que no utilizadas por el estudiantado y el motivo.
En cuanto a las herramientas para crear y gestionar contenido (figura 5), cabe resaltar que la valoración general fue positiva en todos los recursos, aunque existen algunas herramientas mejor valoradas que otras. Las mejor valoradas según el estudiantado (indicando estar "muy satisfecho") son los recursos para hacer presentaciones en línea (72.8\%), el editor de videos $(72.3 \%)$, los procesadores de texto (67.7\%), los formularios en línea (67\%) y la elaboración de presentaciones (66.7\%). Entre las herramientas peor valoradas ("muy insatisfecho" o "insatisfecho") se encuentran aquellas para la elaboración de líneas del tiempo (34.6\%), edición gráfica (15.7\%), presentaciones animadas (13.2\%) y hojas de cálculo (12.5\%), entre otras.

Al igual que en los casos anteriores, las herramientas para compartir y aprender con los demás usuarios fueron valoradas de manera muy positiva en general (ver figura 6). Concretamente, los recursos mejor valorados fueron el sistema de gestión de aprendizaje (aula vir-

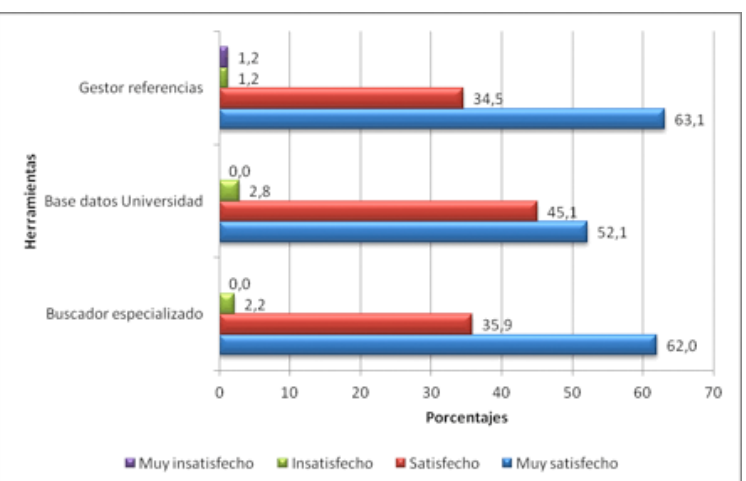

Figura 4. Valoración de las herramientas para buscar y gestionar la información.

tual), donde el $82.5 \%$ señala estar "muy satisfecho"; el correo (74.7\%); el almacenamiento de archivos (72.8\%) y el canal de video (65.1\%). Similar valoración obtienen tanto los recursos para la elaboración de contenidos de forma colaborativa (62.2\%), como los debates virtuales (62.9\%). La herramienta peor valorada por el estudiantado fue el organizador de actividades en línea (Symbaloo), respecto al cual el 12.8\% manifestó estar "muy insatisfecho" o "insatisfecho", aunque cabe resaltar que el restante $87.2 \%$ dijo estar "muy satisfecho" o "satisfecho".

Adicionalmente, se solicitó al estudiantado indicar el grado de dificultad de las herramientas utilizadas. Como se observa en la figura 7, la mayoría de los recursos fueron considerados "fáciles" o "muy fáciles" a la hora de utilizarlos, especialmente, el correo electrónico $(97.9 \%)$, los recursos para la elaboración de documentos colaborativos (93.3\%), la creación de nubes de palabras (93.2\%), el aula virtual $(92.7 \%)$ y el foro para el debate virtual (91\%). Entre las herramientas consideradas como "muy difíciles" o "difíciles" se encuentran 


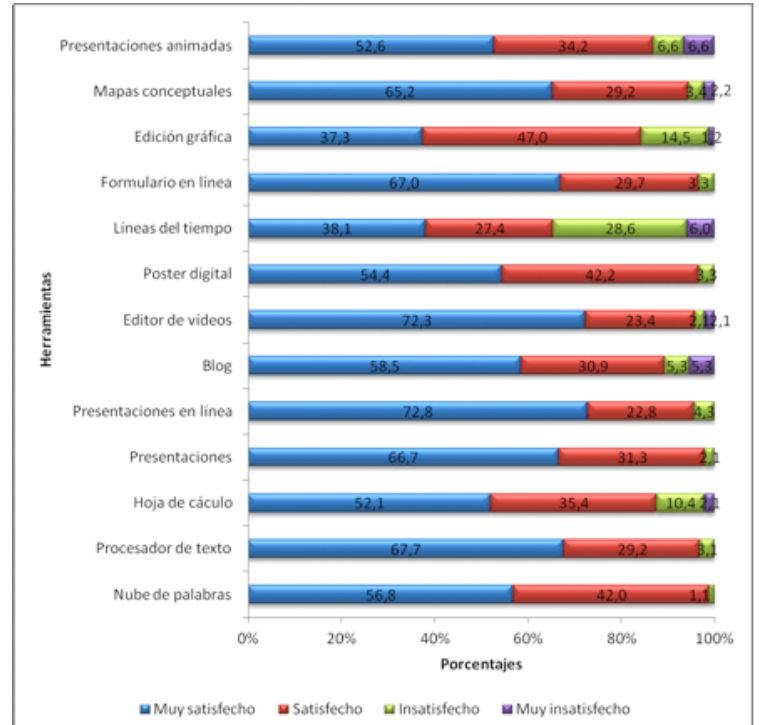

Figura 5. Valoración de las herramientas para crear y gestionar el contenido

aquellas para la elaboración de presentaciones animadas $(71.1 \%)$, la base de datos de la UNA (64.8\%), las hojas de cálculo (56.5\%), el organizador de actividades en línea y las líneas del tiempo (estas dos últimas con un $45.5 \%$ y $42.8 \%$ respectivamente).

\section{Previsión de uso de las diferentes herramientas uti- lizadas}

En cuanto a la previsión de uso de las distintas herramientas (figura 8), el estudiantado anticipó, en cierta medida, la utilización de los recursos para buscar y gestionar la información en el ámbito profesional (32.03\%), personal (26.0\%) o ambos (29.2\%). Tan solo el $12.5 \%$ indicó que no utilizaría este tipo de herramientas en ninguno de los ámbitos señalados.

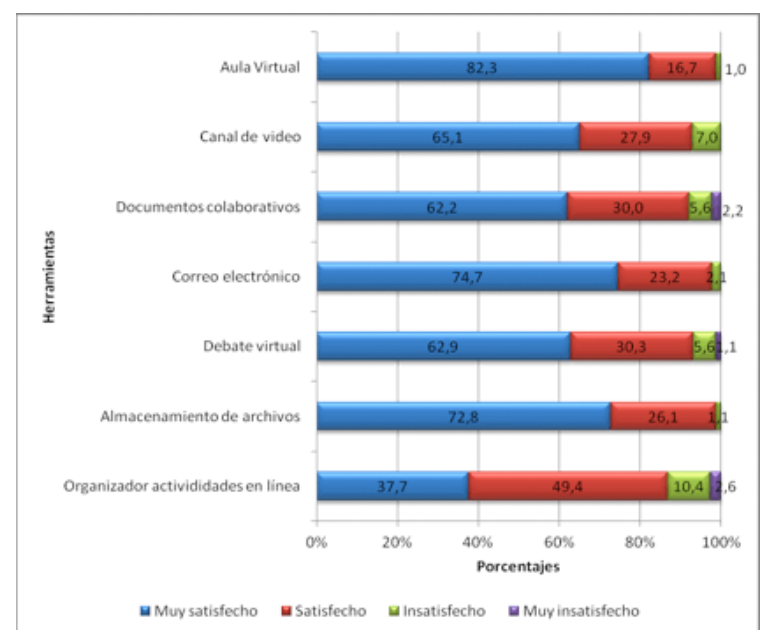

Figura 6. Valoración de las herramientas para compartir y aprender con los demás usuarios.

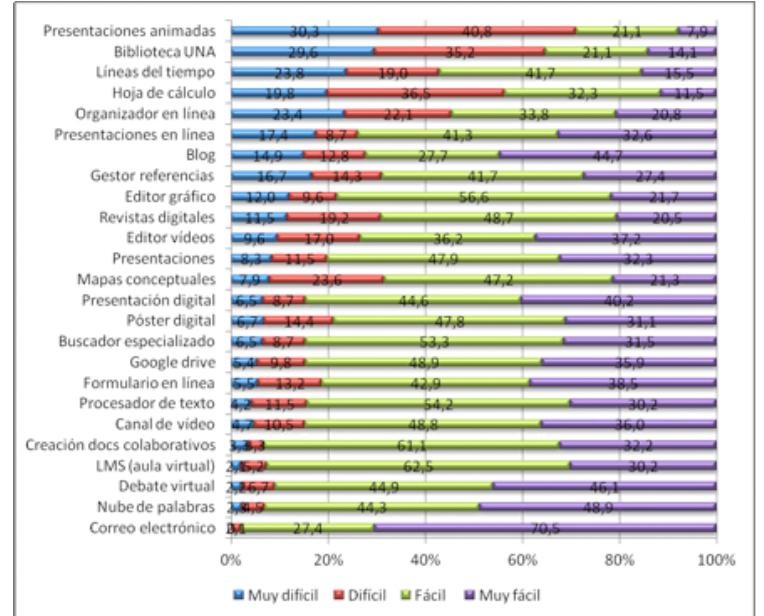

Figura 7. Grado de dificultad de las herramientas utilizadas por el estudiantado.

Respecto a las herramientas para crear y gestionar el contenido, la mayoría de participantes (40.6\%) consideró que las utilizaría en el ámbito profesional; en ambos, tanto profesional como personal (39.6\%). Por el contrario, el $19.8 \%$ apuntó a que solo las usaría en el ámbito personal. Cabe destacar que nadie manifestó que no las utilizaría.

Por último, en relación a aquellos recursos para compartir y aprender de las demás personas, el 51.0\% del estudiantado previó utilizarlos en el ámbito profesional, seguido del uso en ambos ámbitos, profesional y personal (34.4\%). En cambio, el 12,5\% de las personas participantes pronosticó su uso solo en el ámbito personal, y, finalmente, tan solo el $2.1 \%$ no anticipó su uso en ninguno de los ámbitos señalados.

\section{Desarrollo del PLE del estudiantado}

Después de discutir sobre el concepto de PLE, sus componentes y la importancia para el aprendizaje a lo largo de la vida, la percepción que se generó en el estudiantado sobre el desarrollo de sus PLE, específicamente, con respecto al conocimiento y uso de las herramientas vistas durante el curso, fue muy positiva. En este sentido, el $97.9 \%$ indicó haber desarrollado de modo efectivo su PLE, mientras que el $2.1 \%$ restante consideró no haberlo hecho.

En cuanto al nivel de satisfacción con el curso en general, como se observa en la figura 9, el estudiantado declaró estar "muy satisfecho" o "satisfecho" en un $99 \%$, mientras que el restante $1 \%$ manifestó indiferencia; cabe resaltar que nadie indicó estar "insatisfecho" o "muy insatisfecho".

En la tabla 1, se observan algunas de las justificaciones realizadas por el estudiantado, referentes al grado de satisfacción indicado. 
DesarRollo del ENTORNO PERSONAL DE APRENDIZAJE:

VALORACIÓN DE UNA EXPERIENCIA CON ESTUDIANTES UNIVERSITARIOS

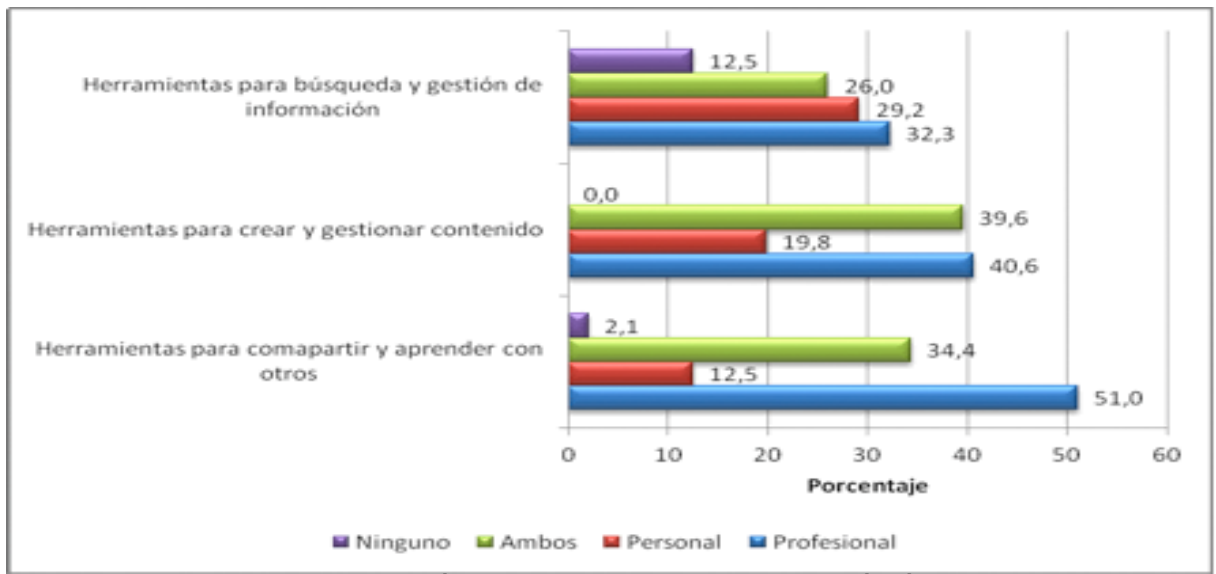

Figura 8. Previsión de uso de herramientas según ámbitos.

\section{Discusión y conclusiones}

El presente estudio ha puesto de manifiesto que el estudiantado de primer año de la carrera de Orientación (promociones 2015 y 2016), en el momento de ingresar a la universidad, tiene muy poco conocimiento de ciertas herramientas, especialmente, de la web 2.0. La excepción la constituyen las redes sociales, cuyo uso no está asociado a procesos de aprendizaje en la mayoría de los casos Estos niveles básicos de competencias tecnológicas pueden ser un impedimento en el desarrollo de los PLE (Anderson, como se citó en Adell, 2009). Coincidiendo estos resultados con los de investigaciones realizadas con muestras similares (Castañeda \& Soto, 2010; García-Martínez \& González-Sanmamed, en prensa), ha quedado expuesto que, además de las redes sociales, las herramientas que más se utilizan en una primera instancia son aquellas destinadas a buscar información (aunque no sean recursos especializados), así como para crear contenido. Las herramientas para compartir y aprender con los demás son las menos utilizadas por el estudiantado.
Este aspecto se ha tener en cuenta de cara al desarroIlo del PLE, ya que, según Siemens (2004), unos de los principios del conectivismo tiene que ver con estar conectados a través de nodos y fuentes de información.

Por otro lado, queda evidenciado que el estudiantado ha adquirido cierto potencial en cuanto al uso de las TIC a lo largo del curso. Este debería ser retomado en la formación de los diferentes cursos de la carrera, ya que si bien se está ofreciendo en la malla curricular un curso específico donde se desarrolla el PLE, la falta de tradición en la integración de las TIC en la disciplina es un obstáculo que impide la adquisición de nuevas competencias (Nogueira-Pérez et al. 2005).

El estudiantado valora muy positivamente las herramientas vistas a lo largo del curso, tanto aquellas para la gestión y búsqueda de información como las de creación de contenido. Este aspecto puede verse afectado por el carácter novedoso de ciertos recursos, así como por la posibilidad de realizar tareas más atractivas que las desarrolladas sin el uso de las TIC, como es el caso de póster digitales, mapas conceptua-
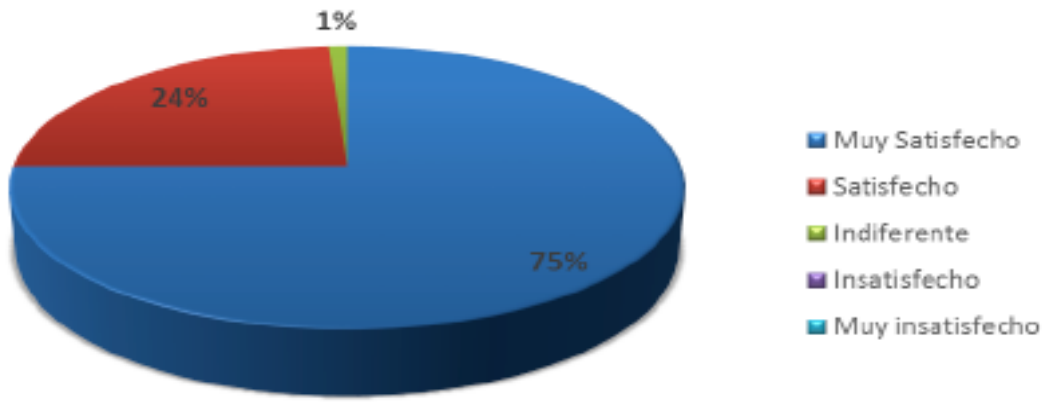

Figura 9. Grado de satisfacción con el curso en general 
Tabla 1. Justificación del grado de satisfacción con el curso en términos generales.

\begin{tabular}{|c|c|}
\hline Grado de satisfacción & Justificación \\
\hline Muy satisfecho & $\begin{array}{l}\text { - "He mejorado notablemente mi nivel de búsqueda de información, } \\
\text { puedo realizar las tareas de manera más creativa y sobre todo com- } \\
\text { partir la información con otros" } \\
\text { - "He logrado utilizar muchas herramientas que no conocía, ya no me } \\
\text { da miedo la tecnología y seguiré utilizándola en lo académico y lo } \\
\text { personal" } \\
\text { - "Había llevado otros cursos sobre Tics, pero no conocía muchas } \\
\text { herramientas de la web 2.0, especialmente el blog que lo voy a se- } \\
\text { guir utilizando después del curso" }\end{array}$ \\
\hline Satisfecho & $\begin{array}{l}\text { - "Realmente el ámbito tecnológico me costaba bastante y he desar- } \\
\text { rollado habilidades con respecto a esto que no consideraba capaz, } \\
\text { entonces esto ha tenido repercusiones a nivel personal ya que pue- } \\
\text { do realizar trabajos académicos con mayor facilidad y poder comu- } \\
\text { nicarme mejor con grupos de trabajo, incluso a distancia" } \\
\text { - "Se aprende de herramientas muy útiles para la universidad y como } \\
\text { futuros profesionales" }\end{array}$ \\
\hline Indiferente & $\begin{array}{l}\text { - "No me gusta la tecnología, aunque hay muchas herramientas que } \\
\text { son muy útiles" }\end{array}$ \\
\hline
\end{tabular}

les, líneas del tiempo, blogs, entre otros, coincidiendo con lo señalado por otras investigaciones (Castañeda \& Soto, 2010; Marín et al., 2014). Por último, el estudiantado muestra igualmente una valoración positiva de las herramientas para compartir información y aprender con otros usuarios, en especial, a través del aula virtual donde se compartían los enlaces de los diferentes trabajos realizados.

En cuanto al grado de dificultad que han encontrado en el uso de las distintas herramientas, cabe destacar que la mayoría del colectivo discente señala que le ha resultado fácil. Esto demuestra ciertas competencias tecnológicas, especialmente, si se tiene en cuenta que muchas de las herramientas que han sido utilizadas provienen de una metodología que otorga mayor peso a la investigación y al descubrimiento, donde se aplican los conocimientos previos y los adquiridos durante el curso.

En cuanto a la previsión de uso de los recursos empleados, cabe remarcar que el estudiantado proyecta su utilización tanto en el ámbito personal como profesional. Este aspecto es sumamente importante, sobre todo si tenemos en cuenta que el uso a nivel personal incluye el componente académico y está estrechamente vinculado con el desarrollo del PLE y el aprendizaje flexible y abierto (Salinas, 2013). Dicho de otro modo, la inclusión de los recursos mencionados en el ámbito personal tiene que ver con la descentralización de un aprendizaje dirigista hacia un proceso donde el estudiantado sea el protagonista de su propio desarrollo. Igual de importante es la previsión de utilización de las herramientas como futuros profesionales en orientación, ya que las demandas del uso y dominio de software especializado en materia de orientación vocacional y profesional son inminentemente necesarias de desarrollar (Pantoja \& Campoy, 2001).

La valoración que el estudiantado realiza del curso en general es muy positiva, coincidiendo con otros estudios (Marín et al., 2014), aspecto que como se ha dicho es beneficioso en términos de atracción del estudiantado. Sin embargo, el reto se presenta con respecto a la incorporación de herramientas tecnológicas en la formación que se brinde en los siguientes cursos. Estos tienen como desafío la vinculación de las TIC al quehacer educativo. Para ello se deberá sortear el obstáculo de la falta de formación especializada en materia de tecnología educativa en orientación, tal como indica Nogueira-Pérez et al. (2005).

La percepción que el estudiantado tiene sobre el desarrollo de su PLE, al finalizar el curso, es muy positiva. Así lo manifiestan algunos participantes al indicar que "he mejorado notablemente mi nivel de búsqueda de información, puedo realizar las tareas de manera más creativa y sobre todo compartir la información con otros", donde se refleja parte de la esencia del PLE. Este aspecto va ligado a la importancia de que el es- 
tudiantado crezca en conciencia sobre el propio concepto, su estructura y del aprendizaje que se genera (Castañeda \& Adell, 2013).

\section{Referencias}

Adell, J. (2009). Sobre entornos personales de aprendizaje. Universitat Jaume I. Recuperado de http://aula.infd. edu.ar/aula/archivos/repositorio/500/711/Entornos Personales de Aprendizaje J Adell.pdf

Adell, J. \& Castañeda, L. (2010). Los Entornos Personales de Aprendizaje (PLEs): una nueva manera de entender el aprendizaje. En R. Roig Vila \& M. Fiorucci (Eds.), Claves para la investigación en innovación y calidad educativas. La integración de las Tecnologías de la Información y la Comunicación y la Interculturalidad en las aulas (pp. 19-30). Alcoy: Marfil.

Álvarez, M. (1995). Orientación profesional. Barcelona: Cedecs.

Attwell, G. (2007). Personal learning environments-the future of elearning? Elearning Papers, 2(1), 1-7. Recuperado de http://somece2015.unam.mx/recursos/ACC/ PLE future of eLearning\%20.pdf

Bisquerra, R. (Coord.). (2003). Metodología de investigación educativa. Madrid: La Muralla.

Cabero, J. \& Marín, V. (2011). Creación de un entorno personal para el aprendizaje: desarrollo de una experiencia. Edutec-e. Revista electrónica de Tecnología Educativa, 38, 1-13. doi:10.21556/edutec.2011.38.380

Castañeda, L. \& Adell, J. (2011). El desarrollo profesional de los docentes en entornos personales de aprendizaje (PLE). En R. Roig Vila \& C. Laneve (Eds.), La práctica educativa en la Sociedad de la Información: Innovación a través de la investigación (pp. 83-95). Alcoy: Marfil.

Castañeda, L. \& Adell, J. (Eds.). (2013). Entornos Personales de Aprendizaje: claves para el ecosistema educativo en red. Alcoy: Marfil.

Castañeda, L. \& Soto, J. (2010). Building Personal Learning Environments by using and mixing ICT tools in a professional way. Digital Education Review, 18, 9-25. Recuperado de http://revistes.ub.edu/index.php/der/ article/view/11319

Chatti, M. A. (11 de enero de 2013). The Laan Theory [Blog post]. Mohamed Amine Chatti's ongoing research on Knowledge and Learning. Recuperado de http:// mohamedaminechatti.blogspot.com.es/2013/01/thelaan-theory.html

Coomey, M. \& Stephenson, J. (2001). Online learning: it is all about dialogue, involvement, support and control-acording to research. En J. Stephenson (Ed.), Teaching and learning Online: Pedagogies for New Technologies (pp. 37-52). Londres: Kogan Page.

Duffy, T. \& Cunningham, D. (1996). Constructivism: Implications for the design and delivery of instruction. En D. Jonassen (Ed.), Handbook of Research for Educational Communications and Technology (pp. 170-198). Nueva York: Macmillan.
García-Martínez, J. \& González-Sanmamed, M. (en prensa). Entornos personales de aprendizaje de estudiantes universitarios costarricenses de educación: análisis de las herramientas de búsqueda de información. Revista de Investigación Educativa.

Hernández, R., Fernández, C. \& Baptista, P. (2010). Metodología de la investigación. México D.F.: McGraw Hill.

Marín V., Lizana, A. \& Salinas, J. (2014). Cultivando el PLE: una estrategia para la integración de aprendizajes en la universidad. Edutec-e. Revista electrónica de Tecnología Educativa, 47, 1-12. doi:10.21556/edutec.2014.47.127

Muñoz-Carril, P. \& González-Sanmamed, M. (2015). Utilización de las TIC en orientación educativa: Un análisis de las plataformas web en los departamentos de orientación de secundaria. Revista Complutense de Educación, 26(2), 447-465. Recuperado de http://revistas.ucm.es/index.php/RCED/article/view/43396

Nogueira-Pérez, M., Fernández-Sestelo, M., Muradás-López, M. \& Porta-Marín, M. (Septiembre, 2005). El uso de las TIC para la orientación educativa a distancia: Valoraciones de los orientadores de A Coruña. Trabajo presentado en el VIII Congreso Galego-Portugués de Psicopedagoxía, Universidade Do Minho, Braga.

Pantoja, A. \& Campoy, T. (2001). Un modelo tecnológico de orientación universitaria. En L. Villar Angulo (Coord.), La universidad, evaluación educativa e innovación curricular (pp. 95-128). Sevilla: Instituto de Ciencias de la Educación.

Prendes, M., Castañeda, L., Ovelar, R. \& Carrera, X. (2014). Componentes básicos para el análisis de los PLE de los futuros profesionales españoles: en los albores del Proyecto CAPPLE. Edutec-e. Revista electrónica de Tecnología Educativa, 47, 1-15. doi:10.21556/edutec.2014.47.139

Rodríguez, M. (1998). La orientación profesional. I Teoría. Barcelona: Ariel Educación.

Ruiz, J., Sánchez, J. \& Gómez-García, M. (2013). Entornos Personales de Aprendizaje: Situación en la Facultad de Ciencias de la Educación de la Universidad de Málaga. Píxel-Bit. Revista de Medios y Educación, 42, 171181. Recuperado de http://acdc.sav.us.es/ojs/index. $\mathrm{php} /$ pixelbit/article/view/471

Salinas, J. (2013). Enseñanza Flexible y aprendizaje abierto, fundamentos clave de los PLEs. En L. Castañeda \& J. Adell (Eds.), Entornos Personales de Aprendizaje: claves para el ecosistema educativo en red (pp. 53-70). Alcoy: Marfil.

Siemens, G. (2004). Connectivism: A learning theory for the digital age. International Journal of Instructional Technology \& Distance Learning, 2(1). Recuperado de http://www.itdl.org/journal/jan_05/article01.htm

Sobrado, L. \& Cortés, A. (Coord.) (2009). Orientación profesional. Nuevos escenarios y perspectivas. Madrid: Biblioteca Nueva. 
Universidad Nacional de Costa Rica. (2006). Plan de estudios del Bachillerato y Licenciatura de la Carrera de Orientación. Costa Rica: Centro de Investigación y Docencia en Educación. UNA.
Venkatesh, V., Croteau, A. \& Rabah, J. (Enero, 2014). Perceptions of Effectiveness of Instructional Uses of Technology in Higher Education in an Era of Web 2.0. Trabajo presentado en la 47th Hawaii International Conference on System Science, Hawaii. 